\title{
DEVOLUCIÓN DE APORTES AL FONAVI, OTRA DEUDA PÚBLICA OLVIDADA DEL GOBIERNO PERUANO
}

\author{
REFUND OF THE FONAVI CONTRIBUTIONS, ANOTHER PUBLIC DEBT \\ FORGOTTEN BY THE PERUVIAN GOVERNMENT \\ Esteban Marino Avelino Sánchez * \\ Docente Asociado de la Facultad de Ciencias Contables
}

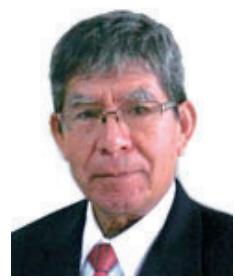

Universidad Nacional Mayor de San Marcos-UNMSM / Lima-Perú

[Recepción: Marzo de 2015/ Conformidad: Abril 2015]

\section{RESUMEN}

La finalidad del Fondo Nacional de Vivienda (FONAVI) fue facilitar el acceso a la casa propia a los trabajadores aportantes, desnaturalizada por la administración deficiente de los gobiernos de turno y destino de los recursos a obras de electrificación, agua y desagüe en los "pueblos jóvenes"; motivó la Ley $\mathrm{N}^{\circ} 29625$ aprobada mediante referéndum, ley de devolución de dinero del FONAVI a los trabajadores que contribuyeron al mismo. El problema es complejo, aquí abordamos solo una arista: la liquidación de los aportes al FONAVI, con las boletas de pago y su actualización, aplicando los factores acumulados de las tasas de interés legal. Los resultados en promedio son sumas razonables, muy superiores a lo dispuesto por el Ministerio de Economía y Finanzas, contraviniendo la Ley 29625. Sin embargo, las sumas actualizadas con la tasa de interés legal de los trabajadores retirados en el año 1990 o en años anteriores, serían menores que la simple dolarización con los tipos de cambio de las fechas en las que se efectuaron los descuentos.

\section{Palabras clave:}

Aportes al FONAVI; porcentajes de aportes al FONAVI; Ley 29625; actualización de aportes al FONAVI; fonavistas.

\begin{abstract}
The purpose of the National Housing Fund (FONAVI) was to facilitate access to home ownership to contributing workers, denatured mismanagement of successive governments and allocation of resources to works of electrification, water and sewer for urban shantytowns, motivated the law No. 29625 passed by referendum, so that the FONAVI law refunds money to the workers who contributed to it. The problem is complex; here we address only an edge: the liquidation of contributions to FONAVI, with paycheck stubs and updates, using the accumulated factors of legal interest rates. The results are reasonable amounts on average, much higher than what was ordered by the Ministry of Economy and Finance, in contravention of Law 29625. However, the amounts updated with the legal interest rate of workers retired in 1990 or in previous years, would be lower than simple dollarization with the exchange rates of the dates on which the discounts were made.
\end{abstract}

\section{Keywords:}

Contributions to FONAVI; percentages of contributions to FONAVI; Law 29625; updating of contributions to FONAVI; fonavistas.

\footnotetext{
* Magíster en Contabilidad con mención en Auditoría. Contador Público y Economista Colegiado. Email: estebanavelino@gmail.com ** Colaborador: Jaime Bernabé Torres Ramírez. Docente Contratado de la Facultad de Ciencias Contables.

*** Colaborador: Richard Tipe Torvisco. Docente Contratado de la Facultad de Ciencias Contables.
} 


\section{INTRODUCCIÓN}

La devolución de los aportes al FONAVI a valores actualizados es otro problema de la deuda pública interna olvidada, impuesta por el gobierno de Morales Bermúdez y olvidada por sus sucesores. La importancia del tema elegido se justifica porque es un problema de actualidad nacional que afecta a algunos millones de ex aportantes al FONAVI, principalmente, de la tercera edad.

Como la mayor parte de la deuda pública interna olvidada, los aportes al FONAVI destinados a fines diferentes al de su creación, tiene poca atención de los intelectuales; para este artículo, se encontraron dos antecedentes bibliográficos: el Semanario Hildebrandt en sus trece. Año 5, $\mathrm{N}^{\circ} 233,16$ de enero de 2015 y el capítulo 12 del libro Manual de Cálculo de Intereses, sexta edición año 2011, en ambos casos en forma somera. El presente artículo tampoco es un estudio minucioso; sin embargo, postula fundamentar la liquidación de los descuentos para el fondo y su actualización de la deuda de cada fonavista.

Por tanto, el objetivo de este artículo es contribuir a determinar el monto a devolver a cada fonavista conforme a ley, mediante un caso práctico. Por ello, la investigación tiene justificación, puede tener una utilidad práctica. El fondo nacional de vivienda, mal gestionado, puede tambien ser estudiado desde otros puntos de vista: jurídico, implicancias macroeconómicas previstas y frustradas, como de sus efectos en las finanzas públicas.

Seguidamente, exponemos la naturaleza de la contribución al FONAVI, la metodología aplicada, la Ley 29625 y normas complementarias, los porcentajes de contribución al FONAVI, la devolución esperada y disposiciones del Gobierno y; un caso práctico sobre liquidación de los aportes al FONAVI y su actualización, conforme a ley.

\section{NATURALEZA DE LA CONTRIBUCIÓN OBLIGATORIA AL FONAVI}

En la constitución de algunos Estados la contribución tributaria, en sentido genérico, incluye los impuestos. En México, Reyes y Nájera (2006) dicen: "En el campo de las ciencias de las finanzas públicas; así como, por la mayoría de las doctrinas sobre el Derecho Tributario, las contribuciones o tributos se apilan en tres secciones: Impuestos, Contribuciones Especiales y Derechos" ${ }^{1}$ Las aportaciones obligatorias del trabajador-empleador por concepto de seguridad social, para beneficiar a los grupos sociales aportantes son casos ilustrativos de las contribuciones. Los autores mencionados, nos recuerdan los principios de tributación de Adam Smith, entre otros el de justicia: "los súbditos de cada Estado deben contribuir al sostenimiento del gobierno en una proporción lo más cercana posible a sus respectivas capacidades"2, en proporción a sus ingresos.

En el Perú, el artículo $19^{\circ}$ de la Constitución Política, señala: "las universidades, institutos superiores y demás centros educativos constituidos conforme a la legislación en la materia, gozan de inafectación de todo impuesto directo o indirecto que afecte a los bienes, actividades y servicios propios de su finalidad educativa y cultural"; sobre los aportes al FONAVI, de cuenta propia, la Universidad San Martín de Porres sostiene dos condiciones: 1) que se trata de un impuesto y 2) que afecta a los bienes, actividades y servicios propios de la finalidad educativa y cultural de las universidades.

La misma universidad nos recuerda la Norma II del Título Preliminar del Código Tributario sobre los conceptos impuesto y contribución: "Impuesto es el tributo cuyo cumplimiento no origina una contraprestación directa a favor del contribuyente por parte del Estado" y, "contribución es el tributo cuya obligación tiene como hecho generador beneficios 
derivados de la realización de obras públicas o de actividades estatales". De lo cual se desprende que el aporte al FONAVI, respecto al empleador representa un impuesto, ya que no tiene ningún beneficio de parte del Estado.

Alega, la SUNAT no puede calificar con base al enunciado del Decreto Legislativo $\mathrm{N}^{\circ} 771$ clasificar al FONAVI como contribución, cuando desde el punto de vista jurídico representa para el empleador un impuesto. "en derecho las instituciones se identifican en razón de su esencia y no en función al nombre que se les asigne, ya que lo contrario llevaría admitir que el nombre que se les atribuye prima sobre el contenido jurídico". El aporte al FONAVI creado por el Decreto Ley $\mathrm{N}^{\circ} 22591$ y leyes modificatorias, en lo que respecta al empleador constituye un impuesto y no una contribución, está dentro de la inafectación señalada en el artículo $19^{\circ}$ de la Constitución Política del Perú.

La Resolución del Tribunal Fiscal N 523-4-97, del 16 de mayo de 1998, dice el vocal informante, para el caso de autos la Ley $\mathrm{N}^{\circ} 26233$, vigente del 2 de noviembre de 1993 hasta el 31 de julio de 1995, regulaba la contribución al FONAVI; de la cual se desprende que, independientemente del destino que el Estado disponga de los recursos recaudados por concepto del FONAVI, respecto del empleador, en rigor la contribución al FONAVI grava la planilla de remuneraciones de los trabajadores dependientes, siendo así que el empleador se encuentra afecto al tributo sin que exista, respecto del mismo, actuación del Estado que le produzca un beneficio.

De acuerdo con la doctrina y definición contenida en el código tributario, la contribución al FONAVI de cuenta propia no califica como una contribución sino como un impuesto, su pago no genera ninguna contraprestación del Estado ni beneficio para el empleador. Por ello, el Tribunal Fiscal resolvió:

1) Revocar la Resolución de Intendencia $N^{\circ} 015-4-$ 05227/SUNAT, del 29 de febrero de 1996 dejándose sin efecto la cobranza de la deuda impugnada;

2) Disponer que la presente resolución constituye jurisprudencia de observancia obligatoria, de acuerdo a lo dispuesto en el artículo $154^{\circ}$ del código tributario deberá ser publicada en el Diario Oficial El Peruano.

\section{METODOLOGÍA}

En forma concisa comentamos la doctrina y jurisprudencia sobre la naturaleza de la contribución al FONAVI, la ley No 29625 (Ley de devolución de dinero del FONAVI a los trabajadores que contribuyeron al mismo) y normas complementarias, los porcentajes de contribución al FONAVI, las liquidaciones de aportes al FONAVI y su actualización realizadas por la Asociación Nacional de Fonavistas, y, desarrollo de un caso práctico.

Técnicas utilizadas: observación, cálculos y análisis de información procesada.

La actualización con las tasas de interés legal efectivas se realizó en la hoja de cálculo y se utilizaron todos los decimales. Si verifica los cálculos con la información que aparece a la vista puede haber diferencias no significativas.

\section{LEY No 29625 Y NORMAS COMPLEMENTARIAS}

Conforme al artículo $1^{\circ}$ se devuelve a: "Todos los trabajadores que contribuyen al FONAVI, el total actualizado de sus aportes que fueron descontados de sus remuneraciones"; así como los aportes de sus respectivos empleadores, $(. .$.$) debidamente actuali-$ zados. Conforme al artículo $2^{\circ}$ se dispone las liquidaciones de aportaciones, de acuerdo a lo señalado en el artículo $1^{\circ}$, y conformar una Cuenta Individual por cada Fonavista. Para efecto de las actualizaciones del valor de las contribuciones se aplicará el interés legal con tasa efectiva, vigente de junio de 1979 hasta el día y mes que se efectúe la liquidación de la Cuenta Individual.

$\mathrm{Su}$ Reglamento, aprobado por Decreto Supremo $\mathrm{N}^{\circ}$ 006-2012-E (13/01/2012), omite las pautas de actualización aludidas en la Ley 29625 . El artículo $9^{\circ}$ dice: "La Comisión, a través de la Secretaría Técnica, podrá contratar servicios de terceros especializados para el mejor cumplimiento de sus funciones", lo que, también, se estaría omitiendo. Contrariando la Ley 29625, el Decreto Supremo No 016-2014-EF (18/01/2014), en el artículo $12^{\circ}$, propone otra forma de liquidación de los aportes al FONAVI, y la actualización de los mismos, aplicando el procedimiento siguiente: 
Monto a devolver $=$ CPMA $x$ VPM.

\section{Dónde:}

CPMA = Cantidad de periodos mensuales aportados, entre julio de 1979 y agosto de 1998, que han sido verificados.

$\mathrm{VPM}=$ Valor de un periodo mensual (cantidad fija, S/.10.00).

\section{PORCENTAJES DE LA CONTRIBUCIÓN AL FONAVI}

Los porcentajes de aportes al FONAVI se compilan en la tabla $\mathrm{N}^{\circ} 01$, de la que se omiten las tasas de contribución facultativa de trabajadores independientes y contribución obligatoria de las empresas constructoras de viviendas financiadas con recursos del FONAVI y de proveedores de bienes y servicios utilizados por dichas empresas; así mismo se excluye el aporte obligatorio del Estado, distinto a la contribución que le correspondió como empleador.

Se observa, de marzo de 1992 a diciembre de 1996, un aumento a $9 \%$ sobre la planilla de remune- ración mensual. Sin embargo, el empleador como el trabajador, conforme a ley, algunos meses en tramos diferentes no aportaron; lo que revela la regulación legal arbitraria de los aportes al FONAVI en la década del noventa, lo que da lugar a cuestionamientos jurídicos como la Resolución del Tribunal Fiscal citada líneas arriba.

\section{DEVOLUCIÓNESPERADAYDISPOSICIONES DEL GOBIERNO}

En la tabla $\mathrm{N}^{\circ} 02$ se resume información de la devolución esperada de siete fonavistas, los cálculos lo habría realizadola Asociación Nacional de Fonavistas de los Pueblos del Perú, conforme a ley. En la misma tabla se observa la suma máxima, dispuesta por el Ministerio de Economía y Finanzas: S/, 1,870.00 (187 meses $x$ S/. 10.00 cada mes), con lo cual se transgrede el artículo $2^{\circ}$ de la Ley 29625. Según la Asociación Nacional de Fonavistas 360,000 fonavistas habrían fallecido en los 16 años de lucha por la devolución de sus aportes al FONAVI.

Tabla No 01:

Contribuciones al FONAVI de julio de 1979 a agosto de 1998

\begin{tabular}{|l|c|c|c|c|}
\hline \multicolumn{1}{|c|}{ Norma Legal } & Vigencia & Empleador & Trabajador & Total \\
\hline D. L. 22591 y D. L. 22845 & Jul. 1979 a Oct. 1988 & 4,0 & 0,5 & 4,5 \\
\hline D. Leg. 497 & Nov. 1988 a Feb. 1992 & 5,0 & 1,0 & 6,0 \\
\hline DSE No 08-PCM/12 & Mar. 1992 a Dic. 1992 & 8,0 & 1,0 & 9,0 \\
\hline D. L. 25981 & Ene. 1993 a Oct. 1993 & 0,0 & 9,0 & 9,0 \\
\hline Ley 26233 & Nov. 1993 a Jul. 1995 & 6,0 & 3,0 & 9,0 \\
\hline Ley 26504 & Ago. 1995 a Dic. 1996 & 9,0 & 0,0 & 9,0 \\
\hline D. Leg. 870 & Ene. 1997 a Jul. 1997 & 7,0 & 0,0 & 7,0 \\
\hline Leyes 26851 y 26969 & Ago. 1997 a Ago. 1998 & 5,0 & 0,0 & 5,0 \\
\hline
\end{tabular}

Fuente: Legislación Laboral Aplicada (págs. 166-167), donde se acopia los porcentajes de las normas legales indicadas. 
Tabla No 02:

Años de aportación al FONAVI, devolución esperada y disposiciones del Gobierno.

Nuevos Soles

\begin{tabular}{|l|c|c|r|c|}
\hline \multicolumn{1}{|c|}{ Nombres (siete casos) } & Edad & $\begin{array}{c}\text { Periodo de } \\
\text { aportación }\end{array}$ & $\begin{array}{c}\text { Devolución } \\
\text { esperada }\end{array}$ & $\begin{array}{c}\text { Disposición del } \\
\text { Gobierno }\end{array}$ \\
\hline 1. Pedro Sánchez Rojas & 76 años & & $51,314^{*}$ & Suma $\leq 1,870$ \\
\hline 2. Delfio Rincón Feliciano & 65 años & 19 Años & $4,600^{*}$ & Suma $\leq 1,870$ \\
\hline 3. Harvey Chávez Borja & 77 años & 19 Años & 156,910 & Suma $\leq 1,870$ \\
\hline 4. Julián García Torres & 79 años & 19 Años & 49,000 & Suma $\leq 1,870$ \\
\hline 5. Rafael Gallardo Cóndor & 60 años & 18 Años & 30,000 & Suma $\leq 1,870$ \\
\hline 6. Esteban Gonzalo Rojas & 72 años & 18 Años & $80,000^{*}$ & Suma $\leq 1,870$ \\
\hline 7. Francisco Sénez Perlado & 82 años & & 57,700 & Suma $\leq 1,870$ \\
\hline
\end{tabular}

* Sumas que habrían sido descontadas de sus remuneraciones.

Fuente: Hildebrandt en sus trece. Año 5, N² 233, 16 de enero de 2015

\section{LIQUIDACIÓN DE LOS APORTES AL FONAVI: CASO PRÁCTICO}

Antes, cabe señalar el límite de la remuneración mensual afecta; hasta mayo de 1986 fue de ocho Sueldos Mínimos Vitales (D.L. 22591 y su Reglamento), a partir de junio de 1986 hasta 8 Unidades Impositivas Tributarias (D. S. N016-86-VC); excluido la movilidad y refrigerio, como los aguinaldos por Fiestas Patrias y Navidad hasta 1988; sin embargo, a partir de 1989 por Decreto Legislativo 870 los aguinaldos por Fiestas Patrias y Navidad fueron incorporados a la base de cálculo de los aportes FONAVI.

Para el caso práctico, se consideran las boletas de pago de un ex trabajador del Instituto Nacional de Planificación, "disuelto" por el Gobierno de Fujimori; por consideraciones prácticas de dos años, se incluye una Boleta de Remuneraciones para evidenciar la fuente de información. En la tabla $\mathrm{N}^{\circ} 03$ se compilan mensualmente, de enero de 1988 a diciembre de 1989, la remuneración mensual afecta y los aportes al FONAVI del empleador y trabajador, la suma de ambos es el aporte total (AT); complementariamente también la Remuneración Mínima Vital (RMV), con la finalidad de mostrar la relación entre ambos conceptos ya que la RMV también se utiliza para actualizar deudas laborales. Puede observar el lector, en la referida tabla, que los aportes al FONAVI fueron 838,981 intis; ¿cuánto equivale en nuevos soles? Menos de un Nuevo Sol; en la misma tabla veremos que los aportes al FONAVI, en promedio, representaron el 41.6\% de la Remuneración Mínima Vital. 
Tabla No 03:

Aportes al FONAVI de un trabajador del Sector Público en el periodo 1988 - 1989. Intis (se omiten los decimales)

\begin{tabular}{|c|c|c|c|c|c|c|}
\hline Mes/Año & $\begin{array}{l}\text { Remuneración } \\
\text { mensual afecta }\end{array}$ & $\begin{array}{l}\text { Aporte del } \\
\text { empleador }\end{array}$ & $\begin{array}{l}\text { Aporte del } \\
\text { trabajador }\end{array}$ & Aporte total & $\underset{*}{\mathbf{A T}} \underset{\text { / RMV }}{ }$ & RMV \\
\hline Ene. 1988 & 22,930 & 917 & 115 & 1,032 & $46.9 \%$ & 2,200 \\
\hline Feb. 1988 & 22,930 & 917 & 115 & 1,032 & $46.9 \%$ & 2,200 \\
\hline Mar. 1988 & 33,276 & 1,331 & 166 & 1,497 & $42.5 \%$ & 3,520 \\
\hline Abr. 1988 & 32,586 & 1,303 & 163 & 1,466 & $41.7 \%$ & 3,520 \\
\hline May. 1988 & 32,586 & 1,303 & 163 & 1,466 & $41.7 \%$ & 3,520 \\
\hline Jun. 1988 & 32,586 & 1,303 & 163 & 1,466 & $41.7 \%$ & 3,520 \\
\hline Jul. 1988 & 46,802 & 1,872 & 234 & 2,106 & $35.0 \%$ & 6,020 \\
\hline Ago. 1988 & 47,012 & 1,880 & 235 & 2,116 & $35.1 \%$ & 6,020 \\
\hline Set. 1988 & 56,012 & 2,240 & 280 & 2,521 & $16.7 \%$ & 15,050 \\
\hline Oct. 1988 & 56,012 & 2,240 & 280 & 2,521 & $16.7 \%$ & 15,050 \\
\hline Nov. 1988 & 28,006 & 2,240 & 280 & 2,521 & $12.0 \%$ & 21,070 \\
\hline Dic. 1988 & 140,200 & 7,012 & 1,402 & 8,414 & $39.9 \%$ & 21,070 \\
\hline Ene. 1989 & 164,300 & 8,213 & 1,643 & 9,856 & $35.2 \%$ & 28,000 \\
\hline Feb. 1989 & 188,900 & 9,445 & 1,889 & 11,334 & $31.5 \%$ & 36,000 \\
\hline Mar. 1989 & 226,700 & 11,334 & 2,267 & 13,601 & $27.8 \%$ & 49,000 \\
\hline Abr. 1989 & 583,600 & 29,180 & 5,836 & 35,016 & $58.4 \%$ & 60,000 \\
\hline May. 1989 & 405,200 & 20,261 & 4,052 & 24,313 & $28.9 \%$ & 84,000 \\
\hline Jun. 1989 & 717,800 & 35,889 & 7,178 & 43,067 & $39.9 \%$ & 108,000 \\
\hline Jul. 1989 & 678,500 & 33,924 & 6,785 & 40,709 & $29.1 \%$ & 140,000 \\
\hline Ago. 1989 & 987,000 & 49,348 & 9,870 & 59,218 & $33.8 \%$ & 175,000 \\
\hline Set. 1989 & $2,718,100$ & 268,933 & 27,181 & 296,114 & $135.2 \%$ & 219,000 \\
\hline Oct. 1989 & $1,336,200$ & 66,810 & 13,362 & 80,172 & $30.8 \%$ & 260,000 \\
\hline Nov. 1989 & 743,100 & 37,155 & 7,431 & 44,586 & $14.0 \%$ & 318,000 \\
\hline Dic. 1989 & $2,547,300$ & 127,365 & 25,473 & 152,838 & $35.1 \%$ & 435,000 \\
\hline \multicolumn{2}{|c|}{ Total dos años } & 722,419 & 116,563 & 838,981 & $41.6 \%$ & $2,014,760$ \\
\hline
\end{tabular}

* Relación del aporte total con la remuneración mínima vital, porcentajes.

Fuente: Boletas de Remuneraciones mensuales de 24 meses. 


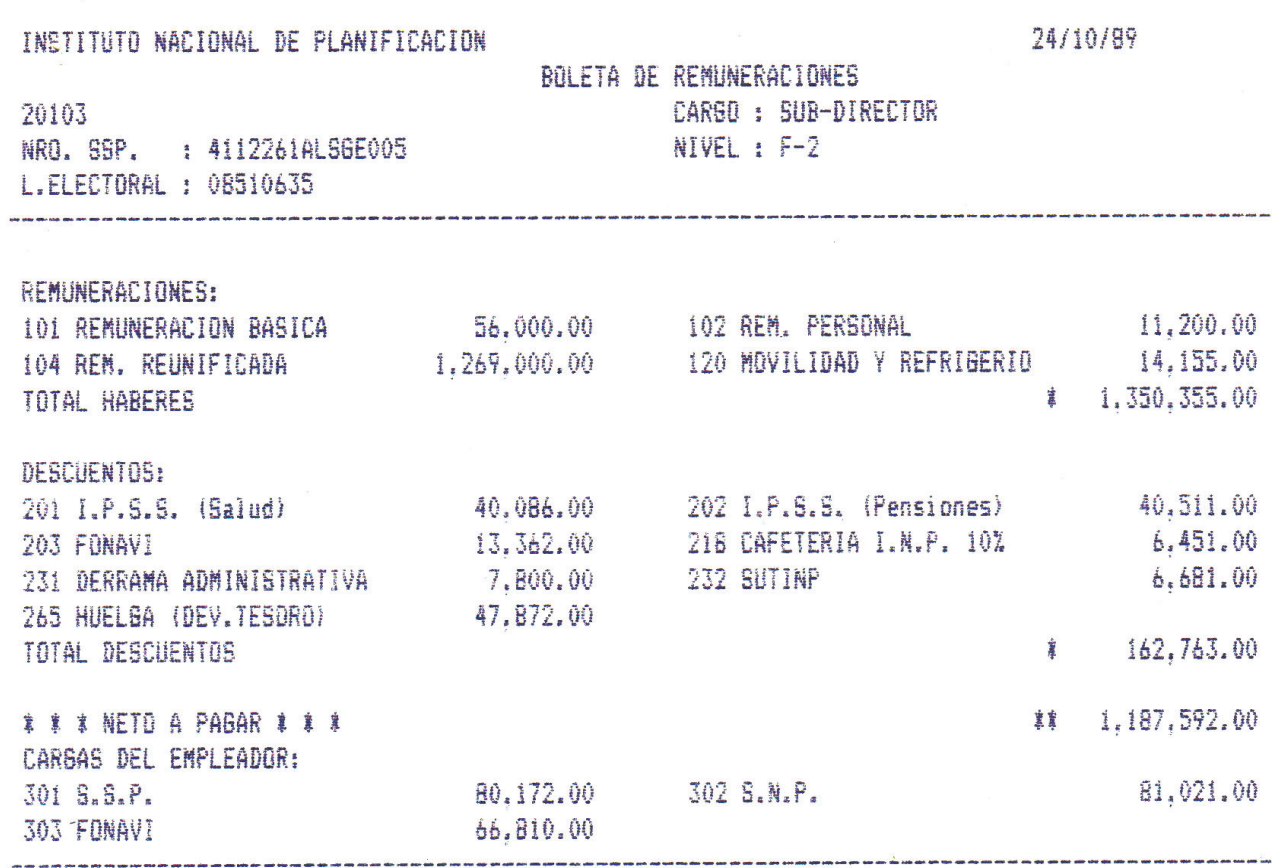

\section{ACTUALIZACIÓN DE LOS APORTES AL FONAVI CONFORME A LEY}

Aunque los cálculos se efectúan con factores acumulados de la tasa de interés legal, se mantiene el principio matemático: $\mathrm{F}=\mathrm{C}(1+\mathrm{i})^{\mathrm{n}}$; en esencia la actualización consiste en el cálculo del valor futuro (simbolizado con la letra F) de sumas de dinero (simbolizadas con la letra C), aportadas mensualmente al FONAVI desde el año 1979 hasta agosto de 1998, con la tasa de interés legal promedio sin truncar los decimales desde la fecha de cada aporte hasta la fecha límite: 31/08/2014 dispuesta por el MEF, se llega sin duda a los mismos resultados obtenidos con los factores acumulados de la misma tasa.

La actualización de los aportes al FONAVI del caso práctico resumido en la tabla $\mathrm{N}^{\circ} 03$, con la metodología del BCRP aplicando factores acumulados (FA), se efectúa en la tabla $\mathrm{N}^{\circ} 04$. En la primera columna de la izquierda se indica el mes y año; en la segunda el factor acumulado correspondiente al último día de cada mes y del 31 de marzo de 1991 (FA: 4678.93335); en la tercera el factor acumulado específico el que resulta de dividir 4678.93335 entre cada uno de los factores acumulados precedentes, cuyo resultado se multiplica sucesivamente por 9.48276 y 6.72410 (FA del 01/04/1991 al 15/09/1992 y del 16/09/1992 al 31/08/2014, respectivamente), como se muestra a continuación:

Enero 1988: (4678.93335/2.71011) x 9.48276 x

$$
6.72410=110085.18151
$$

Febrero 1988: $(4678.93335 / 2.80009)$ x 9.48276 x

$$
6.72410=106547.62928
$$

Diciembre 1989: $(4678.93335 / 114.54962) \mathrm{x}$

$$
9.48276 \times 6.72410=2604.48661
$$

En la penúltima columna de la derecha se consignan los aportes totales mes a mes, provenientes de la tabla $\mathrm{N}^{\circ} 03$, re expresados en nuevos soles con todos los decimales para mantener precisión; como podrá observar la suma de 24 meses de aportación resulta menor a un Nuevo Sol. En la última columna de la derecha se consignan, mes a mes, los aportes al FONAVI actualizados, calculado así:

Enero 1988: $110085.18151 \times 0.001032=S / .113 .59$ Febrero 1988: $106547.62928 \times 0.001032=S / .109 .94$ ...

Diciembre 1989: $2604.48661 \times 0.152838=S / .398 .06$ 
Tabla No 04

Actualización de aportes al FONAVI con interés legal.

Expresado en Nuevos Soles

\begin{tabular}{|c|c|c|c|c|}
\hline Mes y año & $\begin{array}{c}\text { Factores } \\
\text { acumulados * }\end{array}$ & $\begin{array}{c}\text { Factor acumulado } \\
\text { específico }\end{array}$ & Aporte total & $\begin{array}{l}\text { Aporte total } \\
\text { actualizado }\end{array}$ \\
\hline Ene. 1988 & 2.71011 & 110085.18151 & 0.001032 & 113.59 \\
\hline Feb. 1988 & 2.80009 & 106547.62928 & 0.001032 & 109.94 \\
\hline Mar. 1988 & 2.91953 & 102188.69176 & 0.001497 & 153.02 \\
\hline Abr. 1988 & 3.05890 & 97532.75729 & 0.001466 & 143.02 \\
\hline May. 1988 & 3.20992 & 92944.04573 & 0.001466 & 136.29 \\
\hline Jun. 1988 & 3.40770 & 87549.65263 & 0.001466 & 128.38 \\
\hline Jul. 1988 & 3.67452 & 81192.36016 & 0.002106 & 171.00 \\
\hline Ago. 1988 & 3.96224 & 75296.53713 & 0.002116 & 159.29 \\
\hline Set. 1988 & 4.44746 & 67081.64914 & 0.002521 & 169.08 \\
\hline Oct. 1988 & 5.01137 & 59533.21173 & 0.002521 & 150.06 \\
\hline Nov. 1988 & 5.62507 & 53038.08686 & 0.002521 & 133.69 \\
\hline Dic. 1988 & 6.90822 & 43186.66042 & 0.008414 & 363.37 \\
\hline Ene. 1989 & 8.48408 & 35165.03277 & 0.009856 & 346.59 \\
\hline Feb. 1989 & 10.21428 & 29208.41716 & 0.011334 & 331.05 \\
\hline Mar. 1989 & 13.12910 & 22723.79304 & 0.013601 & 309.07 \\
\hline Abr. 1989 & 16.73960 & 17822.58544 & 0.035016 & 624.08 \\
\hline May. 1989 & 21.51653 & 13865.75583 & 0.024313 & 337.12 \\
\hline Jun. 1989 & 27.43358 & 10875.10093 & 0.043067 & 468.36 \\
\hline Jul. 1989 & 35.26222 & 8460.69678 & 0.040709 & 44.43 \\
\hline Ago. 1989 & 45.32490 & 6582.31902 & 0.059218 & 389.79 \\
\hline Set. 1989 & 57.78923 & 5162.60471 & 0.296114 & $1,528.72$ \\
\hline Oct. 1989 & 73.37754 & 4065.86200 & 0.080172 & 325.97 \\
\hline Nov. 1989 & 102.13363 & 2921.10396 & 0.044586 & 130.24 \\
\hline Dic. 1989 & 114.54962 & 2604.48661 & 0.152838 & 398.06 \\
\hline $\begin{array}{c}\text { Mar (31). } \\
1991\end{array}$ & 4678.93335 & & & \\
\hline & & Total dos años & 0.838981 & $7,464.19$ \\
\hline Set (15). 1992 & 9.48276 & \multicolumn{2}{|c|}{ (del 01/04/1991 al 15/09/1992) } & \\
\hline Ago (31).2014 & 6.72410 & \multicolumn{2}{|c|}{ (del 16/09/1992 al 31/08/2014) } & \\
\hline
\end{tabular}

* Desde el 26 de agosto de 1985 al 31 de marzo de 1991, último día de cada mes.

Fuente: Manual de Cálculo de Intereses (tomo II, p. 25-26), $3^{\circ}$ Edición año 2000. Tasa de interés legal (y factores acumulados), del portal electrónico de la SBS.

Elaboración propia. 


\section{ANÁLISIS}

La contribución al FONAVI del caso práctico, de enero de 1988 a diciembre de 1989, actualizada conforme a ley asciende a $S / .7464 .19$, un promedio mensual de S/.311.01, comparado con los S/. 10.00 dispuesto ilegalmente por el MEF la diferencia, en esta casuística, es más de $S / .300 .00$ por mes, deuda interna que el gobierno peruano contraviniendo la Ley no pagará.

Si se actualiza, mes a mes, con la remuneración mínima vital el monto resultante es mayor; por ejemplo, de los aportes de enero de 1988: 1032 intis entre 2200 intis (aportes al FONAVI y RMV del mismo mes y año), luego por 750 nuevos soles resulta S/.351.82. En general, si la antigüedad de la deuda derivada de una relación laboral es anterior a 1989 resulta apropiado la remuneración mínima vital para su actualización, porque debido a la hiperinflación de los años ochenta mientras más antigua sea la deuda la pérdida de su poder adquisitivo no es compensada con la sobre ganancia real obtenida con interés legal entre abril de 1991 y el 15 de setiembre de 1992, periodo en el cual la tasa de interés legal $($ TAMN + 2) fue muy alta respecto a la inflación.

Cabe recordar la hiperinflación habida en el Perú desde 1976 hasta 1990, periodo en el cual las tasas de interés legal fueron muy pequeñas frente a la inflación; de acuerdo con la información presentada en el libro Manual de Cálculo de Intereses tomo I, hasta junio de 1982 mientras la inflación superaba el 60\% anual la tasa de interés legal fue 5\% nominal anual; la tasa de interés legal real acumulada del periodo 1981 a agosto de 1985 fue $-90 \%$, de setiembre de 1985 a noviembre de 1988 de $-89 \%$, de diciembre de 1988 a marzo de 1991 de $-81 \%$ y de abril de 1991 al 15 setiembre de 1992 de 299\% (Avelino, 2000, pp. 174-184).

Por ello en el Perú, las actualizaciones de sumas de dinero en unidades monetarias fuera de circulación son cuestiones controvertidas respecto al indicador económico referente y la metodología utilizada, pasa el tiempo y el deudor simplemente no paga; ejemplos la devolución de los aportes al FONAVI, el pago de los bonos de la Reforma Agraria, etc. La dolarización de la deuda, la dolarización más intereses con la tasa de los del tesoro del Gobierno estadounidense o interés legal son alternativas apropiadas; sin embargo, algunos magistrados, procuradores y abogados se oponen tajantemente, quizá porque desconocen los cambios del valor del dinero a través del tiempo, tanto más en los periodos de hiperinflación.

\section{CONCLUSIONES}

1. Contraviniendo la Ley 29625, el Ministerio de Economía y Finanzas (MEF) ha dispuesto la devolución de montos mínimos por concepto de aportes a los fonavistas.

2. La regulación legal de los aportes al FONAVI en la década del noventa fue arbitraria.

3. La circulación de tres unidades monetarias, durante la vigencia del FONAVI, complica las operaciones de cálculo para la devolución al aportante.

4. El monto a devolver al trabajador considerado en el caso práctico es de $S / .311 .00$ por mes frente a S/.10.00 dispuesto ilegalmente por el MEF, la deuda interna olvidada en esta casuística es de $\mathrm{S} / .301 .00$ por mes correspondiente al trabajador del caso práctico.

\section{REFERENCIAS BIBLIOGRÁFICAS}

1. AVELINO, E. (1998) Legislación Laboral Aplicada. $2^{\circ}$ Edición. Lima - Perú. Editorial San Marcos.

2. AVELINO, E. (2000) Manual de Cálculo de Intereses. $3^{\circ}$ Edición, Tomos I y II. Lima - Perú. Editorial San Marcos.

3. Ley 29625 (2010) Ley de devolución de dinero del FONAVI a los trabajadores que contribuyeron al mismo, del 7 de diciembre del año 2010 y normas reglamentarias y complementarias.

4. Plutón Editores SAC (2015) Semanario Hildebrandt en sus trece. Año 5, $\mathrm{N}^{\circ} 233,16$ de enero de 2015.

5. RENDÓN, J. (1992) Legislación Laboral, Régimen de la actividad privada, Volumen 1. Lima - Perú. Editorial Tarpuy. 
6. REYES, M. y NÁJERA, A. (2006). Derecho Fiscal Apuntes. Tecnológico de Estudios Superiores del Oriente del Estado de México. Recuperado de http://www.tesoem.edu.mx/alumnos/cuadernillos/2006.003.pdf

7. SBS: Portal electrónico, tasas de interés legal. www.sbs.gob.pe
8. Tribunal Fiscal (1998). Resolución del Tribunal Fiscal N 523-4-97, de fecha 16 de mayo de 1998. Recuperado de https://www.mef.gob.pe/contenidos/tribu_fisc/jurisprude/acuer_sala/1997/ resolucion/523-4-97.pdf 